\title{
Learning and Near-Optimal Control of Underactuated Surface Vessels With Periodic Disturbances
}

\author{
Yinyan Zhang, Shuai Li, Senior Member, IEEE, and Jian Weng
}

\begin{abstract}
In this paper, we propose a novel learning and near-optimal control approach for underactuated surface vessels with unknown mismatched periodic external disturbances and unknown hydrodynamic parameters. Given a prior knowledge of the periods of the disturbances, an analytical near-optimal control law is derived through approximation of the integraltype quadratic performance index with respect to the tracking error, where the equivalent unknown parameters are generated online by an auxiliary system that can learn the dynamics of the controlled system. It is proved that the state differences between the auxiliary system and the corresponding controlled underactuated surface vessel are globally asymptotically convergent to zero. Besides, the approach theoretically guarantees asymptotic optimality of the performance index. The efficacy of the method is demonstrated via simulations based on the real parameters of an underactuated surface vessel.
\end{abstract}

Index Terms-Learning and near-optimal control, underactuated surface vessel, mismatched periodic disturbances, auxiliary system.

\section{INTRODUCTION}

$\mathbf{C}$ ONTROLLING underactuated surface vessels (USVs) is a challenging issue due to the nonlinearity of the system model and the fact that they own three degrees of freedom but only two control inputs are available [1], [2]. In practice, autonomous USVs are widely adopted to various missions, such as ocean surveillance and rescue, for which a fundamental task is to automatically track a given reference trajectory with high accuracy. Thus, investigations on the problem are of both theoretical and practical significance.

In recent years, many control methods have been proposed for solving the control problem of USVs. For example, via a global state transformation, Dong and Guo [3] proposed time-varying control laws to achieve the global stabilization. Inspired by Chen et al. [4], Zhang and Li [5] proposed a projection neural network approach to deal with the input constraint for the near-optimal control of USVs. Serrano et al. [6] proposed a linear algebra approach for the tracking control of USVs. Yan and Wang [7] proposed a neural-network-based model predictive control method for the tracking control of USVs with simplified dynamics and known system parameters. However, the control laws in [3]-[7] require full knowledge

Y. Zhang is with the College of Cyber Security, Jinan University, Guangzhou 510632, China (email: yyzhang@jnu.edu.cn).

$\mathrm{S}$. Li is with the College of Engineering, Swansea University, Swansea, United Kingdom (email: shuaili@ieee.org). S. Li is the corresponding author.

J. Weng is with College of Information Science and Technology, Jinan University, Guangzhou 510632, China (email: cryptjweng@gmail.com). of the system dynamics. In practice, there exists parameter uncertainty in the model of USVs, where we may only have nominal values of the system parameters. To handle this issue, Zhang et al. [8] proposed an adaptive near-optimal control approach for USVs with a simplified model of hydrodynamic damping effects, where external disturbances were not explicitly considered. It should be noted that external disturbances are unavoidable in the dynamics of USVs, which become more significant when USVs work on the sea, such as waves and ocean currents.

By considering the above factors, sliding-mode control, which is known for its robustness to external disturbances and system uncertainty, was applied to USVs [9]-[13]. For example, Ashrafiuon et al. [10] proposed a sliding-mode tracking control law for USVs, where a first-order sliding surface about the surge tracking error and a second-order sliding surface with respect to the sway tracking error are adopted. Sliding-mode control laws have good robustness to matched disturbances but are limited by the chattering phenomenon in practice, cannot handle mismatched disturbances, and cannot be directly extended to optimal control.

The backstepping technique was also adopted in the control of USVs for which the combinations with the sliding mode control were further proposed [14]-[21]. For example, Zheng and Feroskhan [18] proposed a backstepping control law for USVs subject to input saturation and external disturbances, where the system parameters are assumed to be known and the external disturbance is handled via a nonlinear disturbance observers. Through a proper error transformation in the control design process, the method in [18] can guarantee that the tracking errors are always bounded by prescribed error boundary trajectories. Chen et al. [20] proposed a radial basis function (RBF) neural network based adaptive backstepping control approach for USVs subject to general external disturbance for which the bounded transient tracking errors are theoretically guaranteed. It should be noted that the setting of parameters of RBFs in neural network based control is not straightforward. Zhang and Yang [22] proposed a fault-tolerant control law for USVs subject to model uncertainty and actuator faults, which can guarantee the convergence of tracking errors to an error bound in finite time given that some requirements on the initial states of the controlled USV are satisfied. Chwa [24] proposed a dynamic surface control law for the tracking control of USVs with input and velocity constraints. The dynamic surface control law adopts the idea of backstepping but remedies for the design complexity called the explosion 
of complexity problem, by applying the low-pass filters in the control law design process. Park et al. [23] proposed a neural network-based output feedback control method for the tracking control of underactuated surface vessels subject to unknown system parameters and external disturbances, where bounded tracking errors can be guaranteed. However, similar to the backstepping control laws, in [23], [24], reference trajectories of all the state variables should be generated by a virtual ship, which may hinder their applications in general tasks where we may only have the desired trajectories of vessel positions. While the above works are related to general disturbances, which can only guarantee bounded tracking errors, to our knowledge, the investigation on the control of USVs under periodic disturbances has not been reported.

The optimal control problem of nonlinear systems generally requires solving a Hamilton-Jacobian-Bellman (HJB) equation, which is a partial differential equation. For most nonlinear systems, the analytical solution of the associated HJB equation is difficult or even impossible to find, for which researchers try to find an approximate optimal solution by using methods like adaptive dynamic programming [25]-[31]. The optimal control of nonlinear systems subject to periodic external disturbances is a challenging issue, for which only a few results have been reported [32], [33]. Tang and Gao [32] proposed an optimal control method for nonlinear systems with fully known sinusoidal disturbances and system dynamics, which transforms the original problem into a sequence of nonhomogeneous linear two-point boundary value problems. However, the methods in [32], [33] require that the system parameters are fully known.

Motivated by the above discussions, in this paper, we investigate the optimal tracking control problem of USVs with unknown hydrodynamic parameters and unknown mismatched periodic external disturbances. Particularly, we require that the control law is explicit so as to facilitate the implementation and save computational resources, the tracking errors are globally asymptotically convergent to zero, and the performance index with respect to the tracking error is asymptotically optimal. Owing to the above factors, the results in repetitive control, such as [34]-[39], do not apply. To solve the problem, we first design an auxiliary system which is able to learn the dynamics of the controlled USV through state feedback and parameter adaptation. Then, based on the theoretical analysis on the performance of the auxiliary system, we employe the Taylor expansion based approach to derive an explicit control law, of which some parameters are generated online by the auxiliary system. The main contributions of this work are summarized in the following.

1) To our knowledge, for the first time, a learning and near-optimal control method is proposed for USVs with unknown periodic external disturbances and unknown hydrodynamic parameters.

2) The proposed near-optimal control method for USVs gives an analytical control law, can handle mismatched periodic external disturbances, and does not need solving the HJB equation by numerical methods.

3) Unlike the methods in [14]-[24], the proposed method does not need a virtual ship to generate reference trajec- tories for all the state variables.

4) Theoretical analysis shows that the proposed method can guarantee globally asymptotic convergence of the output tracking error of the controlled USV to zero and asymptotic optimality of the performance index.

The rest of this paper is organized as follows. In Section II, the problem investigated in this paper is formulated. Then, the design and analysis of the proposed learning and near-optimal control scheme is illustrated in Section III, followed by the simulation verification in Section IV. The concluding remarks are given in Section V.

\section{Problem Formulation}

In this section, we present the model of a USV with unknown hydrodynamic parameters and unknown mismatched periodic external disturbances, and formulate the optimal tracking control problem for the system.

\section{A. USV model}

Under external disturbances, a USV actuated by only one propeller and one rudder with diagonal inertia and damping matrices can be modeled as follows [21]:

$$
\begin{aligned}
\dot{x} & =u \cos (\phi)-v \sin (\phi), \\
\dot{y} & =u \sin (\phi)+v \cos (\phi), \\
\dot{\phi} & =r \\
\dot{u} & =\frac{m_{22}}{m_{11}} v r-\frac{d_{u}}{m_{11}} u-\frac{d_{u 2}}{m_{11}}|u| u-\frac{d_{u 3}}{m_{11}} u^{3}+\frac{\tau_{u}}{m_{11}}+\omega_{u}(t), \\
\dot{v}= & -\frac{m_{11}}{m_{22}} u r-\frac{d_{v}}{m_{22}} v-\frac{d_{v 2}}{m_{22}}|v| v-\frac{d_{v 3}}{m_{22}} v^{3}+\omega_{v}(t), \\
\dot{r}= & \frac{m_{11}-m_{22}}{m_{33}} u v-\frac{d_{r}}{m_{33}} r-\frac{d_{r 2}}{m_{33}}|r| r-\frac{d_{r 3}}{m_{33}} r^{3}+\frac{\tau_{r}}{m_{33}} \\
& +\omega_{r}(t),
\end{aligned}
$$

where $(x, y)$ denote the coordinate of the mass center of the vessel in the earth-fixed frame; $\phi$ denotes the yaw angle in the earth-fixed frame; $u, v$, and $r$ denote the surge, sway and yaw velocities, respectively, in the body-fixed frame; $m_{i i}$ with $i=1,2,3$ denote the accurately known inertia parameters; $d_{u}, d_{u i}, d_{v}, d_{v i}, d_{r}$ and $d_{r i}$ with $i=2,3$ denote unknown hydrodynamic damping coefficients; $\tau_{u}$ and $\tau_{r}$ denoting surge force and yaw moment, respectively, are the two control inputs; $\omega_{u}, \omega_{v}$, and $\omega_{r}$ are the mismatched unknown external disturbances.

In this paper, we focus on the case that the external disturbances are periodic, for which we have the following assumption.

Assumption 1: The unknown external disturbances are periodic with known periods, i.e., $\omega_{u}(t)=\omega_{u}\left(t-T_{u}\right), \omega_{v}(t)=$ $\omega_{v}\left(t-T_{v}\right)$, and $\omega_{r}(t)=\omega_{r}\left(t-T_{r}\right)$ where positive constants $T_{u}, T_{v}$, and $T_{r}$ are known. Besides, the external disturbances are with bounded first-order derivatives with respect to time $t$.

To handle the control singularity problem, we define the position of interest of the vessel as $\mathbf{p}_{\mathrm{i}}=\left[x_{\mathrm{i}}, y_{\mathrm{i}}\right]^{\mathrm{T}}$, where

$$
\begin{aligned}
& x_{\mathrm{i}}=x+L \cos (\phi), \\
& y_{\mathrm{i}}=y+L \sin (\phi),
\end{aligned}
$$

where $L>0 \in \mathbb{R}$ is a small constant. 


\section{B. Adaptive Optimal Dynamic Tracking Problem}

In this paper, we are concerned with the optimal dynamic tracking control problem of the USV (1) with the output defined in (2). Specifically, we aim at finding a pair of control laws $\tau_{u}$ and $\tau_{r}$ that can minimize the following performance index in real time under unknown periodic external disturbances and unknown hydrodynamic damping coefficients:

$$
J=\int_{0}^{T}\left(\mathbf{p}_{\mathbf{i}}(t+h)-\mathbf{p}_{\mathrm{d}}(t+h)\right)^{\mathrm{T}} Q\left(\mathbf{p}_{\mathbf{i}}(t+h)-\mathbf{p}_{\mathbf{d}}(t+h)\right) \mathrm{d} h,
$$

where $T>0 \in \mathbb{R}$ is a design coefficient, and $Q \in \mathbb{R}^{2 \times 2}$ is a symmetric and positive definite coefficient matrix of the performance index; $\mathbf{p}_{\mathrm{d}}(t)=\left[x_{\mathrm{d}}(t), y_{\mathrm{d}}(t)\right]^{\mathrm{T}}$ denotes the desired USV position at time instant $t$.

Assumption 2: The desired trajectory function $\mathbf{p}_{\mathbf{d}}(t)$ is bounded and sufficiently smooth with respect to $t$.

With the above description, the adaptive optimal dynamic tracking problem can be written as the following constrained dynamic optimization problem:

$$
\min _{\tau_{u}, \tau_{r}} J
$$

subject to (1),

where $J$ is defined in (3), for which $\mathbf{p}_{\mathbf{i}}$ is defined in (2). The challenges of the problem lie in the following aspects.

1) There are parameter uncertainty and unknown mismatched periodic external disturbances on the controlled USV (1).

2) This is an optimal control problem of nonlinear underactuated systems, for which the analytical solution is difficult to find, and the numerical approximation for the solution of the associated HJB equation is computationally intensive.

\section{Control Design}

In this section, we illustrate the control law design process for solving the above problem. The proposed learning and near-optimal control method contains two parts, i.e., an auxiliary system and an explicit control law, both of which are theoretically analyzed.

\section{A. Auxiliary System}

We design an auxiliary system, which adaptively updates its parameters such that the errors between the states of the auxiliary system and the corresponding states of the USV asymptotically converges to zero. In this way, the auxiliary system learns the dynamics of the controlled USV. Different from the traditional neural network control approaches, we do not require parameter convergence of the auxiliary system to the actual parameters of the controlled USV.
The auxiliary system is described as follows:

$$
\begin{aligned}
\dot{\hat{u}}= & \frac{m_{22}}{m_{11}} v r-\hat{d}_{11} u-\hat{d}_{12}|u| u-\hat{d}_{13} u^{3}+\frac{\tau_{u}}{m_{11}}+\hat{\omega}_{u}(t) \\
& -k_{x}(\hat{u}-u), \\
\dot{\hat{v}}= & -\frac{m_{11}}{m_{22}} u r-\hat{d}_{21} v-\hat{d}_{22}|v| v-\hat{d}_{23} v^{3}+\hat{\omega}_{v}(t) \\
& -k_{x}(\hat{v}-v), \\
\dot{\hat{r}}= & \frac{m_{11}-m_{22}}{m_{33}} u v-\hat{d}_{31} r-\hat{d}_{32}|r| r-\hat{d}_{33} r^{3}+\frac{\tau_{r}}{m_{33}} \\
& +\hat{\omega}_{r}(t)-k_{x}(\hat{r}-r), \\
\dot{\hat{d}}_{11}= & k_{u} u(\hat{u}-u), \\
\dot{\hat{d}}_{12}= & k_{u}|u| u(\hat{u}-u), \\
\dot{\hat{d}}_{13}= & k_{u} u^{3}(\hat{u}-u), \\
\dot{\hat{d}}_{21}= & k_{v} v(\hat{v}-v), \\
\dot{\hat{d}}_{22}= & k_{v}|v| v(\hat{v}-v), \\
\dot{\hat{d}}_{23}= & k_{v} v^{3}(\hat{v}-v), \\
\dot{\hat{d}}_{31}= & k_{r} r(\hat{r}-r), \\
\dot{\hat{d}}_{32}= & k_{r}|r| r(\hat{r}-r), \\
\dot{\hat{d}}_{33}= & k_{r} r^{3}(\hat{r}-r), \\
\hat{\omega}_{u}(t)= & \hat{\omega}_{u}\left(t-T_{u}\right)-k_{\omega}(\hat{u}-u), \\
\hat{\omega}_{v}(t)= & \hat{\omega}_{v}\left(t-T_{v}\right)-k_{\omega}(\hat{v}-v), \\
\hat{\omega}_{r}(t)= & \hat{\omega}_{r}\left(t-T_{r}\right)-k_{\omega}(\hat{r}-r),
\end{aligned}
$$

where $k_{x}, k_{u}, k_{v}, k_{r}$, and $k_{\omega}$ are positive design constants; $\hat{u}, \hat{v}$, and $\hat{r}$ are state variables of the auxiliary system while $\hat{d}_{i j}$ (with $i=1,2,3$ and $j=1,2,3$ ), $\hat{\omega}_{u}, \hat{\omega}_{v}$, and $\hat{\omega}_{r}$ are the learning parameters. Without generality, we set $\hat{\omega}_{u}(t)=0$, $\hat{\omega}_{v}(t)=0$, and $\hat{\omega}_{r}(t)=0$ for $t \leq 0$.

Let $d_{11}=d_{u} / m_{11}, d_{12}=d_{u 2} / m_{11}, d_{13}=d_{u 3} / m_{11}, d_{21}=$ $d_{v} / m_{22}, d_{22}=d_{v 2} / m_{22}, d_{23}=d_{v 3} / m_{22}, d_{31}=d_{r} / m_{33}$, $d_{32}=d_{r 2} / m_{33}$, and $d_{33}=d_{r 3} / m_{33}$. Let $\tilde{u}=\hat{u}-u, \tilde{v}=\hat{v}-v$, $\tilde{r}=\hat{r}-r, \tilde{d}_{i j}=\hat{d}_{i j}-d_{i j}$ with $i=1,2,3$ and $j=1,2,3$, $\tilde{\omega}_{u}=\hat{\omega}_{u}-\omega_{u}, \tilde{\omega}_{v}=\hat{\omega}_{v}-\omega_{v}$, and $\tilde{\omega}_{r}=\hat{\omega}_{r}-\omega_{r}$.

We have the following lemma regarding the learning performance of the proposed auxiliary system (4) for the USV (1).

Lemma 1: If Assumption 1 holds, for the USV (1) and the auxiliary system (4), given that the external disturbance is bounded and the initial states of the USV (1) and the auxiliary system (4) are bounded, we have $\lim _{t \rightarrow+\infty} \tilde{u}(t)=0$, $\lim _{t \rightarrow+\infty} \tilde{v}(t)=0, \lim _{t \rightarrow+\infty} \tilde{r}(t)=0, \lim _{t \rightarrow+\infty} \dot{\tilde{u}}(t)=0$, $\lim _{t \rightarrow+\infty} \dot{\tilde{v}}(t)=0$, and $\lim _{t \rightarrow+\infty} \dot{\tilde{r}}(t)=0$.

Proof: The proof is given in the appendix.

Lemma 1 shows that the auxiliary system (4) can asymptotically reconstruct the dynamics of the USV (1). In other words, through state feedback, the auxiliary system (4) adaptively updates its parameters such that, under the same control inputs, the evolutions of state variables $u, v, r$, and their first-order derivatives are captured. Thus, the control design with respect to the parts about $u, v$, and $r$ with unknown hydrodynamic parameters and unknown external disturbances 


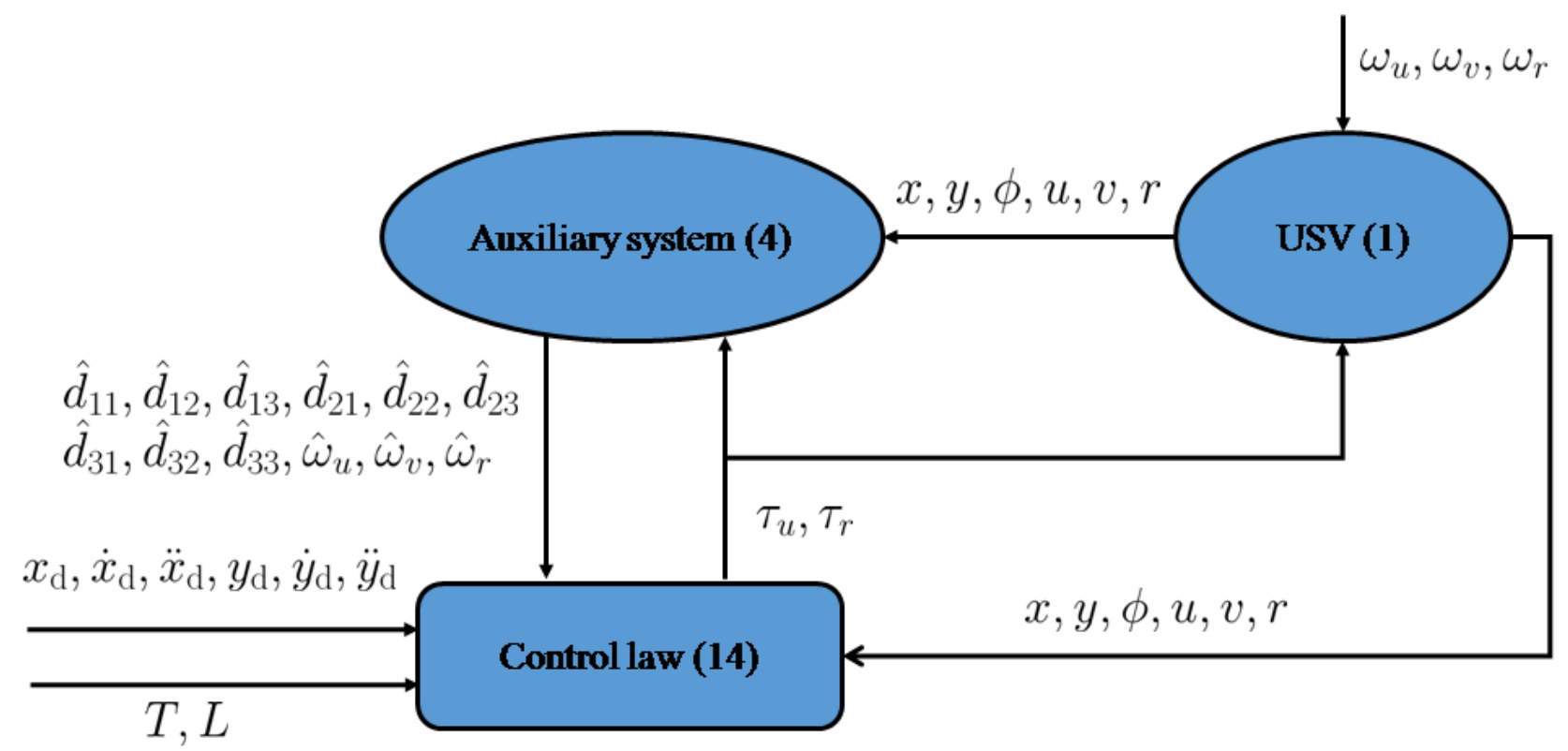

Fig. 1. Block diagram of the proposed method for the learning and near-optimal control (LNOC) of the USV (1) with unknown hydrodynamic parameters and unknown periodic external disturbances, where $x, y, \phi, u, v$, and $r$ are state variables of the USV; $\tau_{u}$ and $\tau_{r}$ are inputs of the USV; $\omega_{u}$, $\omega_{v}$, and $\omega_{r}$ are periodic external disturbances; $x_{\mathrm{d}}, \dot{x}_{\mathrm{d}}, \ddot{x}_{\mathrm{d}}, y_{\mathrm{d}}, \dot{y}_{\mathrm{d}}$, and $\ddot{y}_{\mathrm{d}}$ describe the desired trajectory of the point of interest in the USV, which is defined in (2) with parameter $L ; T$ is the parameter of the performance index (3); $\hat{d}_{i j}$ (with $i=1,2,3$ and $j=1,2,3$ ), $\hat{\omega}_{u}$, $\hat{\omega}_{v}$, and $\hat{\omega}_{r}$ are output of the auxiliary system, which reconstruct the dynamics of the USV (1).

can be performed via the online updated parameters of the auxiliary system (4).

\section{B. Explicit Control Law}

In the following, we design explicit near-optimal control law according to Lemma 1 for the case that $t \rightarrow+\infty$, i.e., the difference between the auxiliary system (4) and the USV (1) approaches zero. With the auxiliary system (4), when $t \rightarrow$ $+\infty$, for the USV (1), we have

$$
\begin{aligned}
\dot{x} & =u \cos (\phi)-v \sin (\phi), \\
\dot{y} & =u \sin (\phi)+v \cos (\phi), \\
\dot{\phi}= & r, \\
\dot{u}= & \frac{m_{22}}{m_{11}} v r-\hat{d}_{11} u-\hat{d}_{12}|u| u-\hat{d}_{13} u^{3}+\frac{\tau_{u}}{m_{11}}+\hat{\omega}_{u}(t) \\
= & \hat{f}_{u}+\frac{\tau_{u}}{m_{11}} \\
\dot{v}= & -\frac{m_{11}}{m_{22}} u r-\hat{d}_{21} v-\hat{d}_{22}|v| v-\hat{d}_{23} v^{3}+\hat{\omega}_{v}(t) \\
= & \hat{f}_{v}, \\
\dot{r}= & \frac{m_{11}-m_{22}}{m_{33}} u v-\hat{d}_{31} r-\hat{d}_{32}|r| r-\hat{d}_{33} r^{3}+\frac{\tau_{r}}{m_{33}} \\
& +\hat{\omega}_{r}(t) \\
= & \hat{f}_{r}+\frac{\tau_{r}}{m_{33}},
\end{aligned}
$$

which serves as an equivalent system for the USV (1) at the equilibrium and is employed in the following to design an explicit near-optimal control law for the USV (1). Let

$$
\begin{aligned}
& c_{\phi}=\cos (\phi), \\
& s_{\phi}=\sin (\phi) .
\end{aligned}
$$

From (5) and (2), when $t \rightarrow+\infty$, we have

$$
\begin{aligned}
\dot{x}_{\mathrm{i}}= & u c_{\phi}-v s_{\phi}-L s_{\phi} r \\
\dot{y}_{\mathrm{i}}= & u s_{\phi}+v c_{\phi}+L c_{\phi} r \\
\ddot{x}_{\mathrm{i}}= & \dot{u} c_{\phi}-u s_{\phi} \dot{\phi}-\dot{v} s_{\phi}-v c_{\phi} \dot{\phi}-L c_{\phi} \dot{\phi} r-L s_{\phi} \dot{r} \\
= & \left(\hat{f}_{u}+\frac{\tau_{u}}{m_{11}}\right) c_{\phi}-u s_{\phi} r-\hat{f}_{v} s_{\phi}-v c_{\phi} r-L c_{\phi} r^{2} \\
& -L s_{\phi}\left(\hat{f}_{r}+\frac{\tau_{r}}{m_{33}}\right) \\
= & \hat{f}_{u} c_{\phi}-u s_{\phi} r-\hat{f}_{v} s_{\phi}-v c_{\phi} r-L c_{\phi} r^{2}-L s_{\phi} \hat{f}_{r}+\frac{\tau_{u}}{m_{11}} c_{\phi} \\
& -L s_{\phi} \frac{\tau_{r}}{m_{33}}, \\
\ddot{y}_{\mathrm{i}}= & \dot{u} s_{\phi}+u c_{\phi} \dot{\phi}+\dot{v} c_{\phi}-v s_{\phi} \dot{\phi}-L s_{\phi} \dot{\phi} r+L c_{\phi} \dot{r} \\
= & \left(\hat{f}_{u}+\frac{\tau_{u}}{m_{11}}\right) s_{\phi}+u c_{\phi} r+\hat{f}_{v} c_{\phi}-v s_{\phi} r-L s_{\phi} r^{2}+L c_{\phi}\left(\hat{f}_{r}\right. \\
& \left.+\frac{\tau_{r}}{m_{33}}\right) \\
= & \hat{f}_{u} s_{\phi}+u c_{\phi} r+\hat{f}_{v} c_{\phi}-v s_{\phi} r-L s_{\phi} r^{2}+L c_{\phi} \hat{f}_{r}+\frac{\tau_{u}}{m_{11}} s_{\phi} \\
& +L c_{\phi} \frac{\tau_{r}}{m_{33}} .
\end{aligned}
$$

Thus, when $t \rightarrow+\infty$,

$$
\begin{aligned}
\dot{\mathbf{p}}_{\mathrm{i}} & =\left[\begin{array}{l}
u c_{\phi}-v s_{\phi}-L s_{\phi} r \\
u s_{\phi}+v c_{\phi}+L c_{\phi} r
\end{array}\right], \\
\ddot{\mathbf{p}}_{\mathrm{i}} & =\left[\begin{array}{l}
\hat{f}_{\tau u} \\
\hat{f}_{\tau r}
\end{array}\right]+S \tau,
\end{aligned}
$$

where $\tau=\left[\tau_{u}, \tau_{r}\right]^{\mathrm{T}}$,

$$
\begin{aligned}
& \hat{f}_{\tau u}=\hat{f}_{u} c_{\phi}-u s_{\phi} r-\hat{f}_{v} s_{\phi}-v c_{\phi} r-L c_{\phi} r^{2}-L s_{\phi} \hat{f}_{r}, \\
& \hat{f}_{\tau r}=\hat{f}_{u} s_{\phi}+u c_{\phi} r+\hat{f}_{v} c_{\phi}-v s_{\phi} r-L s_{\phi} r^{2}+L c_{\phi} \hat{f}_{r},
\end{aligned}
$$


and

$$
S=\left[\begin{array}{cc}
\frac{c_{\phi}}{m_{11}} & -\frac{L s_{\phi}}{m_{33}} \\
\frac{s_{\phi}}{m_{11}} & \frac{L c_{\phi}}{m_{33}}
\end{array}\right] .
$$

Thus, by using the Taylor expansion, when $t \rightarrow+\infty$, we have

$$
\begin{aligned}
\mathbf{p}_{\mathrm{i}}(t+h) & \approx \mathbf{p}_{\mathrm{i}}(t)+h \dot{\mathbf{p}}_{\mathrm{i}}(t)+\frac{h^{2}}{2} \ddot{\mathbf{p}}_{\mathrm{i}}(t) \\
& =\hat{P}_{\mathrm{i}}(t) \mathbf{w}+\frac{h^{2}}{2} S(t) \tau(t), \\
\mathbf{p}_{\mathrm{d}}(t+h) & \approx P_{\mathrm{d}}(t) \mathbf{w},
\end{aligned}
$$

where

$$
\begin{aligned}
\mathbf{w}(h) & =\left[1, h, h^{2} / 2\right]^{\mathrm{T}}, \\
\hat{\mathbf{f}}_{\tau} & =\left[\hat{f}_{\tau u}, \hat{f}_{\tau r}\right]^{\mathrm{T}}, \\
\hat{P}_{\mathrm{i}} & =\left[\mathbf{p}_{\mathrm{i}}, \dot{\mathbf{p}}_{\mathrm{i}}, \hat{\mathbf{f}}_{\tau}\right], \\
P_{\mathrm{d}} & =\left[\mathbf{p}_{\mathrm{d}}, \dot{\mathbf{p}}_{\mathrm{d}}, \ddot{\mathbf{p}}_{\mathrm{d}}\right] .
\end{aligned}
$$

It follows that, when $t \rightarrow+\infty$, the performance index $J$ shown in equation (3) can be approximated as

$$
\begin{aligned}
J \approx & \int_{0}^{T}\left(\hat{P}_{\mathrm{i}} \mathbf{w}(h)+\frac{h^{2}}{2} S \tau-P_{\mathrm{d}} \mathbf{w}(h)\right)^{\mathrm{T}} Q\left(\hat{P}_{\mathrm{i}} \mathbf{w}(h)+\frac{h^{2}}{2} S \tau\right. \\
& \left.-P_{\mathrm{d}} \mathbf{w}(h)\right) \mathrm{d} h \\
= & \int_{0}^{T}\left(\hat{P}_{\mathrm{e}} \mathbf{w}(h)+\frac{h^{2}}{2} S \tau\right)^{\mathrm{T}} Q\left(\hat{P}_{\mathrm{e}} \mathbf{w}(h)+\frac{h^{2}}{2} S \tau\right) \mathrm{d} h \\
= & \int_{0}^{T}\left(\mathbf{w}^{\mathrm{T}}(h) \hat{P}_{\mathrm{e}}^{\mathrm{T}} Q \hat{P}_{\mathrm{e}} \mathbf{w}(h)+h^{2} \mathbf{w}^{\mathrm{T}}(h) \hat{P}_{\mathrm{e}}^{\mathrm{T}} Q S \tau\right. \\
& \left.+\frac{h^{4}}{4} \tau^{\mathrm{T}} S^{\mathrm{T}} Q S \tau\right) \mathrm{d} h \\
= & \int_{0}^{T} h^{2} \mathbf{w}^{\mathrm{T}}(h) \mathrm{d} h \hat{P}_{\mathrm{e}}^{\mathrm{T}} Q S \tau+\int_{0}^{T} \frac{h^{4}}{4} \mathrm{~d} h \tau^{\mathrm{T}} S^{\mathrm{T}} Q S \tau \\
& +\int_{0}^{T} \mathbf{w}^{\mathrm{T}}(h) \hat{P}_{\mathrm{e}}^{\mathrm{T}} Q \hat{P}_{\mathrm{e}} \mathbf{w}(h) \mathrm{d} h \\
= & \frac{T^{5}}{20} \tau^{\mathrm{T}} S^{\mathrm{T}} Q S \tau+\eta^{\mathrm{T}} \hat{P}_{\mathrm{e}}^{\mathrm{T}} Q S \tau \\
& +\int_{0}^{T} \mathbf{w}^{\mathrm{T}}(h) \hat{P}_{\mathrm{e}}^{\mathrm{T}} Q \hat{P}_{\mathrm{e}} \mathbf{w}(h) \mathrm{d} h,
\end{aligned}
$$

where

$$
\hat{P}_{\mathrm{e}}=\hat{P}_{\mathrm{i}}-P_{\mathrm{d}}
$$

and

$$
\eta=\int_{0}^{T} h^{2} \mathbf{w}(h) \mathrm{d} h=\left[\frac{T^{3}}{3}, \frac{T^{4}}{4}, \frac{T^{5}}{10}\right]^{\mathrm{T}} .
$$

Because the decision variable is the input $\tau$, minimizing $T^{5} \tau^{\mathrm{T}} S^{\mathrm{T}} Q S \tau / 20+\eta^{\mathrm{T}} \hat{P}_{\mathrm{e}}^{\mathrm{T}} Q S \tau+\int_{0}^{T} \mathbf{w}^{\mathrm{T}}(h) \hat{P}_{\mathrm{e}}^{\mathrm{T}} Q \hat{P}_{\mathrm{e}} \mathbf{w}(h) \mathrm{d} h$ is equivalent to minimizing

$$
\hat{J}=T^{5} \tau^{\mathrm{T}} S^{\mathrm{T}} Q S \tau / 20+\eta^{\mathrm{T}} \hat{P}_{\mathrm{e}}^{\mathrm{T}} Q S \tau .
$$

Note that $S^{\mathrm{T}} Q S$ is at least positive semidefinite, owing to the fact that $Q$ is symmetric and positive definite. From the definition of $S$ shown in (8), the determinant of $S$ is

$$
\operatorname{det}(S)=\frac{L c_{\phi}^{2}}{m_{11} m_{33}}+\frac{L s_{\phi}^{2}}{m_{11} m_{33}}=\frac{L}{m_{11} m_{33}}>0 .
$$

Because $Q$ is symmetric and positive definite, we have $\operatorname{det}(Q)>0$. It follows that

$$
\operatorname{det}\left(S^{\mathrm{T}} Q S\right)=\operatorname{det}(S) \times \operatorname{det}(Q) \times \operatorname{det}(S)>0 .
$$

Recalling that $S^{\mathrm{T}} Q S \in \mathbb{R}^{2 \times 2}$ is at least positive semidefinite, for which the eigenvalues are not less than 0, i.e., $\lambda_{1}\left(S^{\mathrm{T}} Q S\right) \geq 0$ and $\lambda_{2}\left(S^{\mathrm{T}} Q S\right) \geq 0$. Then, from (12) and the fact that $\operatorname{det}\left(S^{\mathrm{T}} Q S\right)=\lambda_{1}\left(S^{\mathrm{T}} Q S\right) \times \lambda_{2}\left(S^{\mathrm{T}} Q S\right)$, we further have $\lambda_{1}\left(S^{\mathrm{T}} Q S\right)>0$ and $\lambda_{2}\left(S^{\mathrm{T}} Q S\right)>0$. Thus, the matrix $S^{\mathrm{T}} Q S$ is positive definite, and $\hat{J}$ is convex with respect to $\tau$. Then, the learning and near-optimal control law is derived by solving $\partial \hat{J} / \partial \tau=0$, i.e.,

$$
\frac{T^{5} S^{\mathrm{T}} Q S \tau}{10}+S^{\mathrm{T}} Q \hat{P}_{\mathrm{e}} \eta=0,
$$

which yields

$$
\begin{aligned}
\tau & =-\frac{10}{T^{5}}\left(S^{\mathrm{T}} Q S\right)^{-1} S^{\mathrm{T}} Q \hat{P}_{\mathrm{e}} \eta \\
& =-\frac{10}{T^{5}} S^{-1} Q^{-1}\left(S^{\mathrm{T}}\right)^{-1} S^{\mathrm{T}} Q \hat{P}_{\mathrm{e}} \eta \\
& =-\frac{10}{T^{5}} S^{-1} \hat{P}_{\mathrm{e}} \eta .
\end{aligned}
$$

Then, it follows from (13), and the definitions of $S, \hat{P}_{\mathrm{e}}$, and $\eta$ that, the learning and near-optimal control laws for the surge force and yaw moment are

$$
\begin{aligned}
\tau_{u}= & m_{11}\left(-\hat{\omega}_{u}+\hat{d}_{11} u+r v+L r^{2}+\ddot{x}_{\mathrm{d}} c_{\phi}+\hat{d}_{13} u^{3}+\ddot{y}_{\mathrm{d}} s_{\phi}\right. \\
& \left.+\hat{d}_{12} u|u|\right)+\frac{10 m_{11}}{3 T^{2}}\left(x_{\mathrm{d}} c_{\phi}-x c_{\phi}-L-y s_{\phi}+y_{\mathrm{d}} s_{\phi}\right) \\
& +\frac{5}{2 T}\left(\dot{x}_{\mathrm{d}} m_{11} c_{\phi}-m_{11} u+\dot{y}_{\mathrm{d}} m_{11} s_{\phi}\right)-m_{22} r v \\
\tau_{r}= & \frac{m_{33}}{L}\left(\hat{d}_{21} v-\hat{\omega}_{v}-r u+\ddot{y}_{\mathrm{d}} c_{\phi}+\hat{d}_{23} v^{3}-\ddot{x}_{\mathrm{d}} s_{\phi}-L \hat{\omega}_{r}\right. \\
& \left.+L \hat{d}_{31} r+L \hat{d}_{33} r^{3}+\hat{d}_{22} v|v|+L \hat{d}_{32} r|r|\right)+m_{22} u v \\
& -m_{11} u v+\frac{m_{11} m_{33} r u}{L m_{22}}-\frac{5 m_{33}}{2 L T}\left(v-\dot{y}_{\mathrm{d}} c_{\phi}+L r\right) \\
& -\frac{10 m_{33}}{3 L T^{2}}\left(y c_{\phi}-y_{\mathrm{d}} c_{\phi}-x s_{\phi}+x_{\mathrm{d}} s_{\phi}\right)
\end{aligned}
$$

where $s_{\phi}=\sin (\phi)$ and $c_{\phi}=\cos (\phi)$. The information flow in the proposed control structure is shown in Fig. 1.

\section{Theoretical Results}

In this subsection, theoretical results are given about the closed-loop performance of the USV (1) synthesized by the proposed auxiliary system (4) and the LNOC law (13), i.e., (14).

Theorem 1: If Assumption 1 and Assumption 2 hold, the tracking error $\mathbf{e}(t)=\mathbf{p}_{\mathbf{i}}(t)-\mathbf{p}_{\mathrm{d}}(\mathrm{t})$, with $\mathbf{p}_{\mathrm{i}}$ defined in (2), of the USV (1) synthesized by the LNOC law (13), i.e., (14), and the auxiliary system (4) is globally asymptotically convergent to zero.

Proof: From equations (1) and (2), we have

$$
\ddot{\mathbf{p}}_{\mathrm{i}}=\mathbf{f}_{\tau}+S \tau
$$

with $\mathbf{f}_{\tau}=\left[f_{\tau u}, f_{\tau r}\right]^{\mathrm{T}}$, where

$$
\begin{aligned}
f_{\tau u} & =f_{u} c_{\phi}-u s_{\phi} r-f_{v} s_{\phi}-v c_{\phi} r-L c_{\phi} r-L s_{\phi} f_{r}, \\
f_{\tau r} & =f_{u} s_{\phi}+u c_{\phi} r+f_{v} c_{\phi}-v s_{\phi} r-L s_{\phi} r^{2}+L c_{\phi} f_{r}
\end{aligned}
$$


with $f_{u}, f_{v}$ and $f_{r}$ defined as follows:

$$
\begin{aligned}
f_{u} & =\frac{m_{22}}{m_{11}} v r-d_{11} u-d_{12}|u| u-d_{13} u^{3}+\omega_{u}, \\
f_{v} & =-\frac{m_{11}}{m_{22}} u r-d_{21} v-d_{22}|v| v-d_{23} v^{3}+\omega_{v}, \\
f_{r} & =\frac{m_{11}-m_{22}}{m_{33}} u v-d_{31} r-d_{32}|r| r-d_{33} r^{3}+\omega_{r} .
\end{aligned}
$$

Substituting the control law (13) into (15) yields

$$
\ddot{\mathbf{p}}_{\mathrm{i}}=\mathbf{f}_{\tau}-\frac{10}{T^{5}} \hat{P}_{\mathrm{e}} \eta
$$

which, together with equations (11) and (9), gives

$\ddot{\mathbf{p}}_{\mathrm{i}}=\mathbf{f}_{\tau}-\frac{10}{T^{5}}\left(\frac{T^{3}}{3}\left(\mathbf{p}_{i}-\mathbf{p}_{\mathrm{d}}\right)+\frac{T^{4}}{4}\left(\dot{\mathbf{p}}_{\mathrm{i}}-\dot{\mathbf{p}}_{\mathrm{d}}\right)+\frac{T^{5}}{10}\left(\hat{\mathbf{f}}_{\tau}-\ddot{\mathbf{p}}_{\mathrm{d}}\right)\right)$.

Let $\tilde{\mathbf{f}}_{\tau}=\hat{\mathbf{f}}_{\tau}-\mathbf{f}_{\tau}$. With $\mathbf{e}=\mathbf{p}_{\mathrm{i}}-\mathbf{p}_{\mathrm{d}}$ and $\delta=T^{5} \tilde{\mathbf{f}}_{\tau} / 10$, from (18), the closed-loop error dynamics is derived as follows:

$$
\frac{T^{5}}{10} \ddot{\mathbf{e}}+\frac{T^{4}}{4} \dot{\mathbf{e}}+\frac{T^{3}}{3} \mathbf{e}=\delta,
$$

The characteristic equation of (19) is

$$
\frac{T^{5}}{10} \sigma^{2}+\frac{T^{4}}{4} \sigma+\frac{T^{3}}{3}=0
$$

of which the roots are $\sigma=5\left(-T^{4} / 4 \pm\right.$ $\left.\sqrt{T^{8} / 16-2 T^{8} / 15}\right) / T^{5}$. Obviously, both roots are located on the left-hand side of the complex plane. Thus, if $\delta(t) \equiv 0$, e is globally exponentially convergent to zero.

Let $\tilde{f}_{u}=\hat{f}_{u}-f_{u}, \tilde{f}_{v}=\hat{f}_{v}-f_{v}$, and $\tilde{f}_{r}=\hat{f}_{r}-f_{r}$. From (7) and (16), we have

$$
\begin{gathered}
\tilde{f}_{\tau u}=\tilde{f}_{u} c_{\phi}-\tilde{f}_{v} s_{\phi}-L s_{\phi} \tilde{f}_{r}, \\
\tilde{f}_{\tau r}=\tilde{f}_{u} s_{\phi}+\tilde{f}_{v} c_{\phi}+L c_{\phi} \tilde{f}_{r} .
\end{gathered}
$$

Based on equations (17) and (6), According to Lemma 1, we have $\lim _{t \rightarrow+\infty} \tilde{f}_{u}(t)=0, \lim _{t \rightarrow+\infty} \tilde{f}_{v}(t)=0$, $\lim _{t \rightarrow+\infty} \tilde{f}_{r}(t)=0$. It follows that $\lim _{t \rightarrow+\infty} \tilde{f}_{\tau u}(t)=0$ and $\lim _{t \rightarrow+\infty} \tilde{f}_{\tau r}(t)=0$. Consequently, in view of $\delta=T^{5} \tilde{\mathbf{f}}_{\tau} / 10$ and $\mathbf{f}_{\tau}=\left[f_{\tau u}, f_{\tau r}\right]^{\mathrm{T}}$, we further have

$$
\lim _{t \rightarrow+\infty} \delta(t)=0
$$

by which and the bounded-input-bounded-output (BIBO) property [40] we further conclude that e of the closed-loop error dynamics (19) is globally asymptotically convergent to zero. The proof is complete.

Theorem 2: If Assumption 1 and Assumption 2 hold, when $t \rightarrow+\infty$, the LNOC law (13), i.e., (14), aided by the auxiliary system (4), is optimal for the performance index (3) of the USV (1).

Proof: From (10) and the design process of the LNOC law (13), (13) is optimal for the following performance index:

$$
\begin{aligned}
J_{\mathrm{a}}= & \int_{0}^{T}\left(\hat{P}_{\mathrm{i}} \mathbf{w}(h)+\frac{h^{2}}{2} S \tau-P_{\mathrm{d}} \mathbf{w}(h)\right)^{\mathrm{T}} Q\left(\hat{P}_{\mathrm{i}} \mathbf{w}(h)+\frac{h^{2}}{2} S \tau\right. \\
& \left.-P_{\mathrm{d}} \mathbf{w}(h)\right) \mathrm{d} h
\end{aligned}
$$

TABLE I

PARAMETERS OF THE USV AdOPTED IN THE SimUlation

\begin{tabular}{lll}
\hline Parameters & Values & Units \\
\hline$m_{11}$ & $1.2 \times 10^{5}$ & $\mathrm{~kg}$ \\
$m_{22}$ & $1.779 \times 10^{5}$ & $\mathrm{~kg}$ \\
$m_{33}$ & $6.36 \times 10^{7}$ & $\mathrm{~kg}$ \\
$d_{u}$ & $2.15 \times 10^{4}$ & $\mathrm{~kg} / \mathrm{s}$ \\
$d_{u 2}$ & $4.3 \times 10^{3}$ & $\mathrm{~kg} / \mathrm{m}$ \\
$d_{u 3}$ & $2.15 \times 10^{3}$ & $\mathrm{~kg} / \mathrm{m}^{2}$ \\
$d_{v}$ & $1.47 \times 10^{5}$ & $\mathrm{~kg} / \mathrm{s}$ \\
$d_{v 2}$ & $2.94 \times 10^{4}$ & $\mathrm{~kg} / \mathrm{m}$ \\
$d_{v 3}$ & $1.47 \times 10^{4}$ & $\mathrm{~kg} \cdot \mathrm{s} / \mathrm{m}^{2}$ \\
$d_{r}$ & $8.02 \times 10^{6}$ & $\mathrm{~kg} \cdot \mathrm{m}^{2} / \mathrm{s}$ \\
$d_{r 2}$ & $1.604 \times 10^{6}$ & $\mathrm{~kg} \cdot \mathrm{m}^{2}$ \\
$d_{r 3}$ & $8.02 \times 10^{5}$ & $\mathrm{~kg} \cdot \mathrm{m}^{2} \mathrm{~s}$ \\
\hline
\end{tabular}

In view of the definition of the performance index (3), the output (2), and the USV dynamics (1), taking the residual term of the Taylor expansion into account, we have

$$
\begin{aligned}
J= & \int_{0}^{T}\left(\hat{P}_{\mathrm{i}} \mathbf{w}(h)-\tilde{P}_{\mathrm{i}} \mathbf{w}(h)+\frac{h^{2}}{2} S \tau-P_{\mathrm{d}} \mathbf{w}(h)+\nu(t)\right)^{\mathrm{T}} Q \\
& \cdot\left(\hat{P}_{\mathrm{i}} \mathbf{w}(h)-\tilde{P}_{\mathrm{i}} \mathbf{w}(h)+\frac{h^{2}}{2} S \tau-P_{\mathrm{d}} \mathbf{w}(h)+\nu(t)\right) \mathrm{d} h
\end{aligned}
$$

where $\tilde{P}_{\mathrm{i}}=\left[\mathbf{0}, \mathbf{0}, \tilde{\mathbf{f}}_{\tau}\right]$ and $\nu(t)=h^{2} / 2(\ddot{\mathbf{e}}(t+\gamma h)-\ddot{\mathbf{e}}(t))$ with $\mathbf{0} \in \mathbb{R}^{2}$ and $\gamma \in(0,1)$. From the proof of Theorem 1 , we have $\lim _{t \rightarrow+\infty} \tilde{\mathbf{f}}_{\tau}(t)=0$, by which we have $\lim _{t \rightarrow+\infty} \tilde{P}_{\mathrm{i}}(t)=0$. Meanwhile, from Theorem 1 , we have $\lim _{t \rightarrow+\infty} \mathbf{e}(t)=0$, by which and Barbalat's lemma [40], we conclude that $\lim _{t \rightarrow+\infty} \ddot{\mathbf{e}}(t)=0$. It follows that, $\lim _{t \rightarrow+\infty} \nu(t)=0$. Let the difference between the above two performance indices be denoted by $J_{\mathrm{e}}=J-J_{\mathrm{a}}$. From the above analysis, we have $\lim _{t \rightarrow+\infty} J_{\mathrm{e}}(t)=\lim _{t \rightarrow+\infty}\left(J(t)-J_{\mathrm{a}}(t)\right)=$ $\lim _{t \rightarrow+\infty}\left(J_{\mathrm{a}}(t)-J_{\mathrm{a}}(t)\right)=0$. Thus, when $t \rightarrow+\infty$, the control law (13), i.e., (14), aided by the auxiliary system (4), is optimal for the performance index (3) of the USV (1). The proof is complete.

\section{Simulation VAlidation}

In this section, simulation results based on the parameters of a USV are given to validate the performance of the proposed method. Specifically, we consider a monohull surface vessel equipped with one propeller and one rudder, which is a typical USV. The length of the vessel is $38 \mathrm{~m}$ and its mass is $1.18 \times 10^{5}$ $\mathrm{kg}$. The parameters of the model (1) for the USV is obtained from [41] and are shown in Table I. In terms of the output parameter defined in (2), we set $L=0.01 \mathrm{~m}$. The parameters of the performance index is set to $T=0.2 \mathrm{~s}$ and $Q=I$, where $I$ is a 2-by-2 identity matrix. The parameters of the auxiliary system (4) is set to $k_{x}=50, k_{u}=k_{v}=k_{\omega}=5$, and $k_{r}=100 k_{u}=500$ in view of the large difference between $m_{33}$ and $m_{11}$. The desired trajectory is given as follows:

$$
\begin{aligned}
& x_{\mathrm{d}}(t)=100 \sin (0.05 t)+1.2, \\
& y_{\mathrm{d}}(t)=100 \cos (0.05 t)-99 .
\end{aligned}
$$




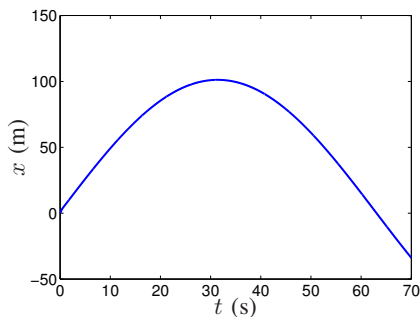

(a) State variable $x$

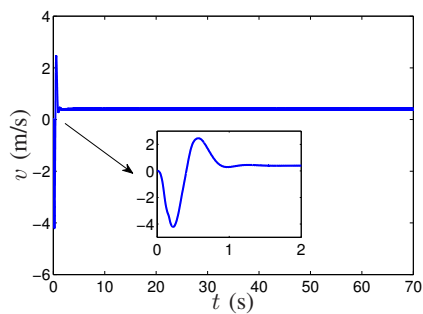

(e) State variable $v$

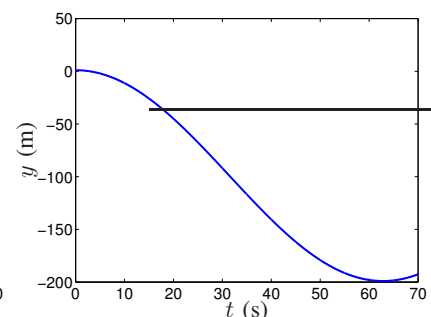

(b) State variable $y$

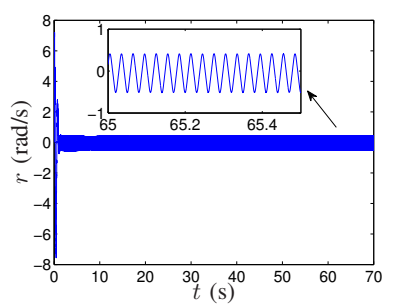

(f) State variable $r$

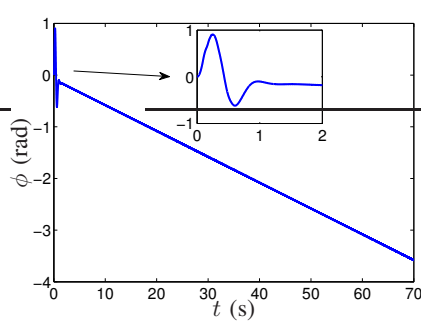

(c) State variable $\phi$

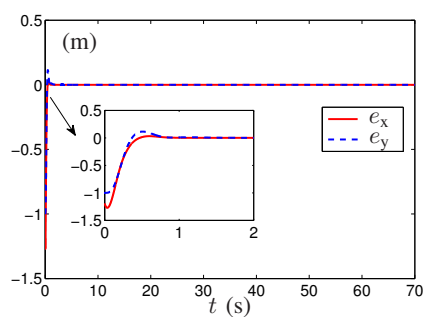

(g) Tracking errors $e_{\mathrm{x}}$ and $e_{\mathrm{y}}$

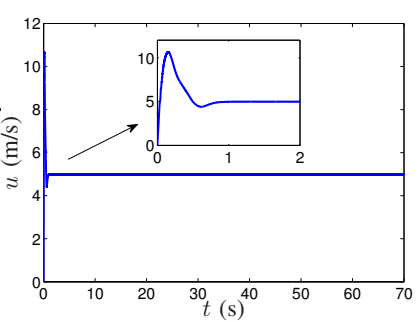

(d) State variable $u$

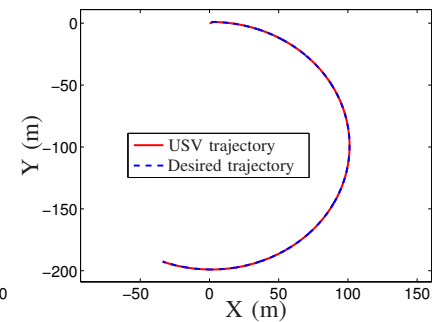

(h) USV trajectories

Fig. 2. Data profiles regarding the state variables and tracking performance of the USV during the tracking control process of the USV synthesized by the proposed control law (14) with saturation, i.e., (22), and the proposed auxiliary system (4) under mismatched periodic external disturbances shown in equation (21) and hydrodynamic parameter uncertainty for the desired trajectory (20).

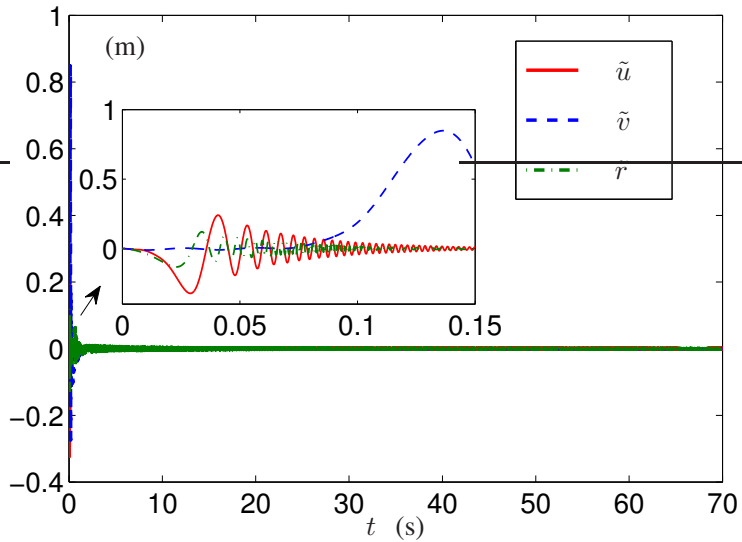

(a) $\tilde{u}, \tilde{v}$ and $\tilde{r}$

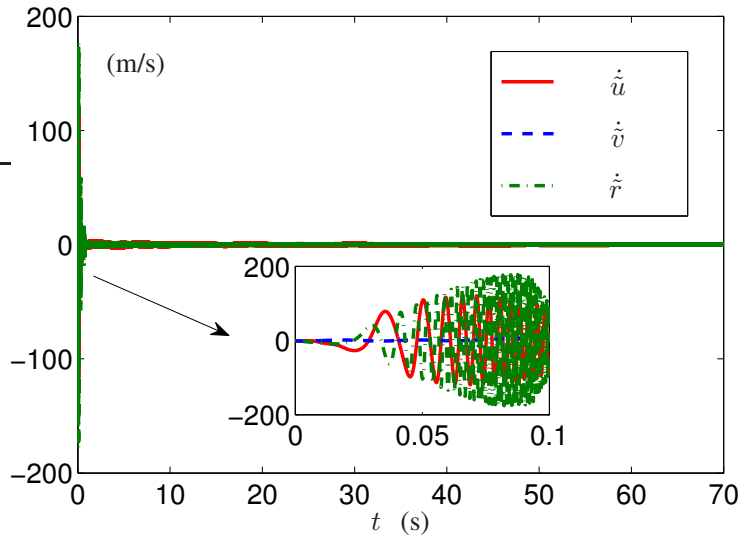

(b) $\dot{\tilde{u}}$, $\dot{\tilde{v}}$ and $\dot{\tilde{r}}$

Fig. 3. Profiles regarding the learning performance of the auxiliary system (4) during the tracking control process of the USV synthesized by the proposed control law (14) with saturation, i.e., (22), under mismatched periodic external disturbances shown in equation (21) and hydrodynamic parameter uncertainty for the desired trajectory (20), where $\tilde{u}=\hat{u}-u, \tilde{v}=\hat{v}-v$, and $\tilde{r}=\hat{r}-r$.

The external periodic disturbances are described as follows:

$$
\begin{aligned}
& \omega_{u}(t)=1.2 \sin \left(\frac{2 \pi t}{T_{u}}+\frac{\pi}{6}\right)+0.4, \\
& \omega_{v}(t)=1.4 \sin \left(\frac{2 \pi t}{T_{v}}+\frac{\pi}{6}\right)+0.2, \\
& \omega_{r}(t)=0.8 \sin \left(\frac{2 \pi t}{T_{r}}+\frac{\pi}{8}\right)+0.5,
\end{aligned}
$$

where the known periods are $T_{u}=0.01 \mathrm{~s}, T_{v}=0.03 \mathrm{~s}$, and $T_{r}=0.05 \mathrm{~s}$.

Without loss of generality, the initial values of the state variables of the USV is set to $x(0)=y(0)=\phi(0)=$ $u(0)=v(0)=r(0)=0$, and accordingly, for the auxiliary system (4), $\hat{u}(0)=\hat{v}(0)=\hat{r}(0)=0$. The initial values of $\hat{d}_{i j}$ with $i=1,2,3$ and $j=1,2,3$ of the auxiliary system (4) are set to randomly generated positive values, while $\hat{\omega}_{u}(0)=\hat{\omega}_{v}(0)=\hat{\omega}_{r}(0)=0$. Considering the actuator constraint, the actual control inputs applied to the USV are set as follows:

$$
\begin{aligned}
\tau_{u \mathrm{a}} & =P_{\mathbb{S}}\left(\tau_{u}\right), \\
\tau_{r \mathrm{a}} & =P_{\mathbb{S}}\left(\tau_{r}\right),
\end{aligned}
$$

where $\tau_{u}$ and $\tau_{r}$ are calculated according to the proposed control law (14) with the parameters updated online via the auxiliary system (4), and $P_{\mathbb{S}}(\cdot)$ denotes the projection operator on the set $\mathbb{S}=\left\{x \mid-5 \times 10^{9} \leq x \leq 5 \times 10^{9}\right\}$. As seen from Fig. $3, \tilde{u}(t), \tilde{v}(t), \tilde{r}(t), \dot{\tilde{u}}(t), \dot{\tilde{v}}(t)$, and $\dot{\tilde{r}}(t)$ asymptotically converge to zero, indicating that the auxiliary system (1) reconstructs the dynamics of the controlled USV, which validates Lemma 1. From Fig. 2, the state variables of the USV are bounded during the control process, and with the aid of the proposed control law (14) with saturation, i.e., (22), and the auxiliary system (1), the tracking errors $e_{\mathrm{x}}=x_{\mathrm{i}}-x_{\mathrm{d}}$ and $e_{\mathrm{y}}=y_{\mathrm{i}}-y_{\mathrm{d}}$ asymptotically converge to zero with fast convergence and low overshooting, which validates Theorem 1. According to Fig. 


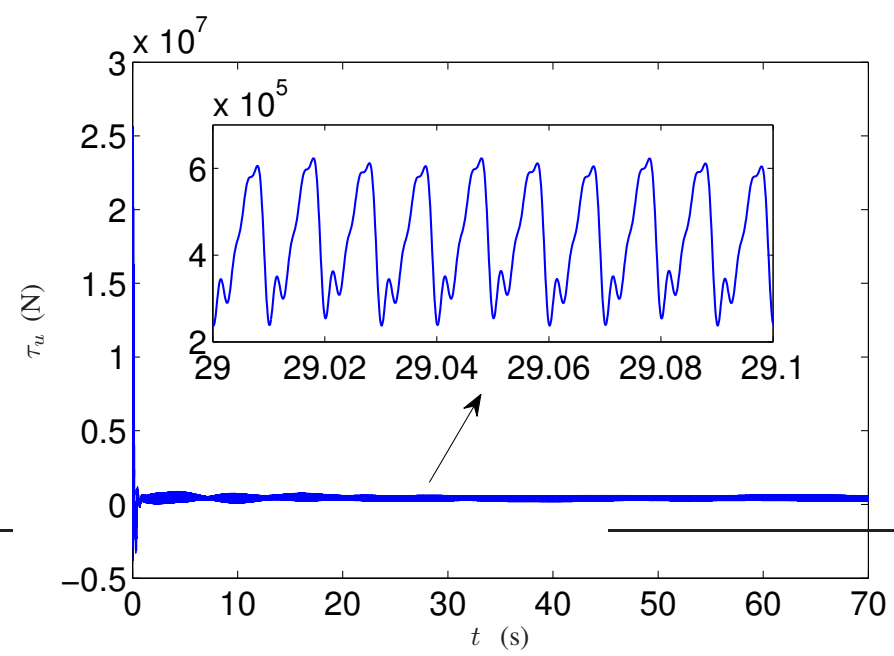

(a) Surge force $\tau_{u}$

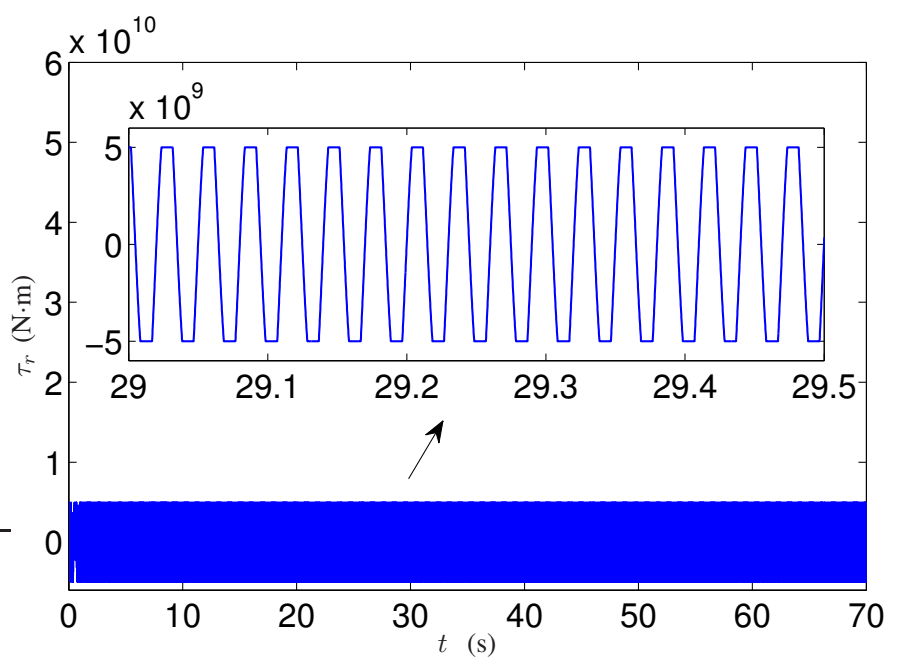

(b) Yaw moment $\tau_{r}$

Fig. 5. Profiles of inputs during the tracking control process of the USV synthesized by the proposed control law (14) with saturation, i.e., (22), under mismatched periodic external disturbances shown in equation (21) and hydrodynamic parameter uncertainty for the desired trajectory (20).

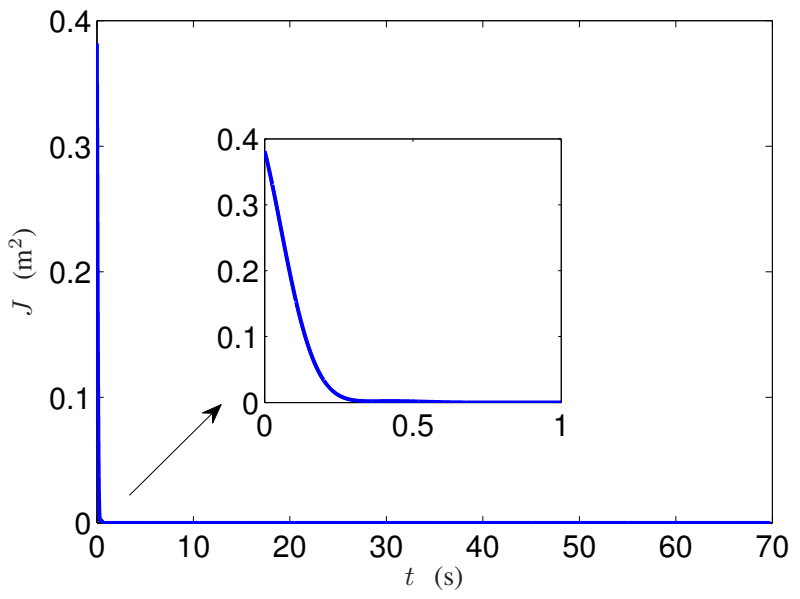

Fig. 4. Profile of performance index $J$ (3) during the tracking control process of the USV synthesized by the proposed control law (14) with saturation, i.e., (22), under mismatched periodic external disturbances shown in equation (21) and hydrodynamic parameter uncertainty for the desired trajectory (20).

4, the performance index $J(t)$ is asymptotically convergent to zero, which validates Theorem 2. As seen from Fig. 5, the control inputs are bounded. Note that the chartering in the control inputs is needed for the purpose of completely eliminating the effects of periodic external disturbances. These results shows the effectiveness of the proposed method for the near-optimal control of USVs (1) under unknown mismatched periodic external disturbances and unknown hydrodynamic parameters.

\section{CONCLusions}

In this paper, for the first time, a novel learning and near-optimal control method has been proposed for USVs with unknown hydrodynamic parameters and unknown mismatched periodic external disturbances. The method consists of an auxiliary system to reconstruct the dynamics of the controlled USV via state feedback, of which the adaptively updated parameters are employed in an explicit control law for the controlled USV. Theoretical analysis has shown that the proposed method can guarantee the global asymptotic convergence of the tracking error to zero and the asymptotic optimality of the performance index for USVs with unknown hydrodynamic parameters and unknown mismatched periodic external disturbances. Simulation results based on the parameters of a USV have validated the performance of the proposed method, including fast tracking error convergence and low overshooting.

\section{APPENDIX}

The proof of Lemma 1 is given as follow.

Proof: Recalling Assumption 3 and that fact that the parameters of the USV are constants, from the USV (1) and the auxiliary system (4), we have

$$
\begin{aligned}
\dot{\tilde{u}} & =-\tilde{d}_{11} u-\tilde{d}_{12}|u| u-\tilde{d}_{13} u^{3}+\tilde{\omega}_{u}(t)-k_{x} \tilde{u}, \\
\dot{\tilde{v}} & =-\tilde{d}_{21} v-\tilde{d}_{22}|v| v-\tilde{d}_{23} v^{3}+\tilde{\omega}_{v}(t)-k_{x} \tilde{v}, \\
\dot{\tilde{r}} & =-\tilde{d}_{31} r-\tilde{d}_{32}|r| r-\tilde{d}_{33} r^{3}+\tilde{\omega}_{r}(t)-k_{x} \tilde{r}, \\
\dot{\tilde{d}}_{11} & =k_{u} u \tilde{u}, \\
\dot{\tilde{d}}_{12} & =k_{u}|u| u \tilde{u}, \\
\dot{\tilde{d}}_{13} & =k_{u} u^{3} \tilde{u} \\
\dot{\tilde{d}}_{21} & =k_{v} v \tilde{v} \\
\dot{\tilde{d}}_{22} & =k_{v}|v| v \tilde{v}, \\
\dot{\tilde{d}}_{23} & =k_{v} v^{3} \tilde{v} \\
\dot{\tilde{d}}_{31} & =k_{r} r \tilde{r} \\
\dot{\tilde{d}}_{32} & =k_{r}|r| r \tilde{r} \\
\dot{\tilde{d}}_{33} & =k_{r} r^{3} \tilde{r} \\
\tilde{\omega}_{u}(t) & =\tilde{\omega}_{u}\left(t-T_{u}\right)-k_{\omega} \tilde{u}, \\
\tilde{\omega}_{v}(t) & =\tilde{\omega}_{v}\left(t-T_{v}\right)-k_{\omega} \tilde{v}, \\
\tilde{\omega}_{r}(t) & =\tilde{\omega}_{r}\left(t-T_{r}\right)-k_{\omega} \tilde{r},
\end{aligned}
$$


from which we further have

$$
\begin{aligned}
\tilde{\omega}_{u}^{2}(t) & -\tilde{\omega}_{u}^{2}\left(t-T_{u}\right) \\
& =\left(\tilde{\omega}_{u}(t)+\tilde{\omega}_{u}\left(t-T_{u}\right)\right)\left(\tilde{\omega}_{u}(t)-\tilde{\omega}_{u}\left(t-T_{u}\right)\right) \\
& =-k_{\omega} \tilde{u}\left(\tilde{\omega}_{u}(t)+\tilde{\omega}_{u}\left(t-T_{u}\right)\right) .
\end{aligned}
$$

Accordingly, we have

$$
\begin{aligned}
& \tilde{\omega}_{v}^{2}(t)-\tilde{\omega}_{v}^{2}\left(t-T_{v}\right)=-k_{\omega} \tilde{v}\left(\tilde{\omega}_{v}(t)+\tilde{\omega}_{v}\left(t-T_{v}\right)\right), \\
& \tilde{\omega}_{r}^{2}(t)-\tilde{\omega}_{r}^{2}\left(t-T_{r}\right)=-k_{\omega} \tilde{r}\left(\tilde{\omega}_{r}(t)+\tilde{\omega}_{r}\left(t-T_{r}\right)\right) .
\end{aligned}
$$

Consider the following Lyapunov candidate function:

$$
\begin{aligned}
V= & \sum_{j=1}^{3} \frac{\tilde{d}_{1 j}^{2}}{2 k_{u}}+\sum_{j=1}^{3} \frac{\tilde{d}_{2 j}^{2}}{2 k_{v}}+\sum_{j=1}^{3} \frac{\tilde{d}_{3 j}^{2}}{2 k_{r}}+\frac{\tilde{u}^{2}}{2}+\frac{\tilde{v}^{2}}{2}+\frac{\tilde{r}^{2}}{2} \\
& +\frac{\int_{t-T_{u}}^{t} \tilde{\omega}_{u}^{2}(\tau) \mathrm{d} \tau}{2 k_{\omega}}+\frac{\int_{t-T_{v}}^{t} \tilde{\omega}_{v}^{2}(\tau) \mathrm{d} \tau}{2 k_{\omega}}+\frac{\int_{t-T_{r}}^{t} \tilde{\omega}_{r}^{2}(\tau) \mathrm{d} \tau}{2 k_{\omega}} .
\end{aligned}
$$

Together with equations (23) and (24), the time derivative of $V$ along the state trajectory of the USV (1) and the auxiliary system (4) is obtained as follows:

$$
\begin{aligned}
\dot{V}= & \sum_{j=1}^{3} \frac{\dot{\tilde{d}}_{1 j} d_{1 j}}{k_{u}}+\sum_{j=1}^{3} \frac{\dot{\tilde{d}}_{2 j} d_{2 j}}{k_{v}}+\sum_{j=1}^{3} \frac{\dot{\tilde{d}}_{3 j} d_{3 j}}{k_{r}}+\dot{\tilde{u}} \tilde{u}+\dot{\tilde{v}} \tilde{v}+\dot{\tilde{r}} \tilde{r} \\
& +\frac{\tilde{\omega}_{u}^{2}(t)-\tilde{\omega}_{u}^{2}\left(t-T_{u}\right)}{2 k_{3}}+\frac{\tilde{\omega}_{v}^{2}(t)-\tilde{\omega}_{v}^{2}\left(t-T_{v}\right)}{2 k_{3}} \\
& +\frac{\tilde{\omega}_{r}^{2}(t)-\tilde{\omega}_{r}^{2}\left(t-T_{r}\right)}{2 k_{3}} \\
= & \tilde{d}_{11} u \tilde{u}+\tilde{d}_{12}|u| u \tilde{u}+\tilde{d}_{13} u^{3} \tilde{u}+\tilde{d}_{21} v \tilde{v}+\tilde{d}_{22}|v| v \tilde{v} \\
& +\tilde{d}_{23} v^{3} \tilde{v}+\tilde{d}_{3} 1 r \tilde{r}+\tilde{d}_{32}|r| r \tilde{r}+\tilde{d}_{33} r^{3} \tilde{r}-\tilde{d}_{11} u \tilde{u} \\
& -\tilde{d}_{12}|u| u \tilde{u}-\tilde{d}_{13} u^{3} \tilde{u}+\tilde{\omega}_{u}(t) \tilde{u}-k_{x} \tilde{u}^{2}-\tilde{d}_{21} v \tilde{v} \\
& -\tilde{d}_{22}|v| v \tilde{v}-\tilde{d}_{23} v^{3} \tilde{v}+\tilde{\omega}_{v}(t) \tilde{v}-k_{x} \tilde{v}^{2}-\tilde{d}_{31} r \tilde{r} \\
& -\tilde{d}_{32}|r| r \tilde{r}-\tilde{d}_{33} r^{3} \tilde{r}_{r} \tilde{\omega}_{r}(t) \tilde{r}-k_{x} \tilde{r}^{2} \\
& -\frac{\left(\tilde{\omega}_{u}(t)+\tilde{\omega}_{u}\left(t-T_{u}\right)\right) \tilde{u}}{2}-\frac{\left(\tilde{\omega}_{v}(t)+\tilde{\omega}_{v}\left(t-T_{v}\right)\right) \tilde{v}}{2} \\
& -\frac{\left(\tilde{\omega}_{r}(t)+\tilde{\omega}_{r}\left(t-T_{r}\right)\right) \tilde{r}}{2} \\
= & -k_{x} \tilde{u}^{2}-k_{x} \tilde{v}^{2}-k_{x} \tilde{r}^{2}+\frac{\left(\tilde{\omega}_{u}(t)-\tilde{\omega}_{u}\left(t-T_{u}\right)\right) \tilde{u}}{2} \\
& +\frac{\left(\tilde{\omega}_{v}(t)-\tilde{\omega}_{v}\left(t-T_{v}\right)\right) \tilde{v}}{2}+\frac{\left(\tilde{\omega}_{r}(t)-\tilde{\omega}_{r}\left(t-T_{r}\right)\right) \tilde{r}}{2} \\
\leq & -k_{x} \tilde{u}^{2}-k_{x} \tilde{v}^{2}-k_{x} \tilde{r}^{2}-\frac{1}{2} k_{\omega} \tilde{u}^{2}-\frac{1}{2} k_{\omega} \tilde{v}^{2}-\frac{1}{2} k_{\omega} \tilde{r}^{2} \\
& 0 .
\end{aligned}
$$

From (25), we have

$$
\begin{aligned}
\ddot{V}= & -\left(2 k_{x}+k_{\omega}\right) \tilde{u} \dot{\tilde{u}}-\left(2 k_{x}+k_{\omega}\right) \tilde{v} \dot{\tilde{v}}-\left(2 k_{x}+k_{\omega}\right) \tilde{r} \dot{\tilde{r}} \\
= & \left(2 k_{x}+k_{\omega}\right) \tilde{u}\left(\tilde{d}_{11} u+\tilde{d}_{12}|u| u+\tilde{d}_{13} u^{3}-\tilde{\omega}_{u}(t)+k_{x} \tilde{u}\right) \\
& +\left(2 k_{x}+k_{\omega}\right) \tilde{v}\left(\tilde{d}_{21} v+\tilde{d}_{22}|v| v+\tilde{d}_{23} v^{3}-\tilde{\omega}_{v}(t)+k_{x} \tilde{v}\right) \\
& +\left(2 k_{x}+k_{\omega}\right) \tilde{r}\left(\tilde{d}_{31} r+\tilde{d}_{32}|r| r+\tilde{d}_{33} r^{3}-\tilde{\omega}_{r}(t)+k_{x} \tilde{r}\right) .
\end{aligned}
$$

Meanwhile, from (25), we also have $0 \leq V(t) \leq V(0)$, $\forall t>0$. Thus, $\tilde{d}_{i j}$ (with $i=1,2,3$ and $j=1,2,3$ ), $\tilde{u}$, $\tilde{v}, \tilde{r}, \tilde{\omega}_{u}, \tilde{\omega}_{v}$, and $\tilde{\omega}_{r}$ are bounded, $\forall t>0$, starting from bounded initial values. It follows that $\ddot{V}$ is bounded, by which we conclude that $\dot{V}$ is uniformly continuous. Besides, the above analysis also shows that that $V(t)$ is lower bounded and $\dot{V}(t)$ is negative semi-definite. Thus, according to Barbalat's lemma [40], we have $\lim _{t \rightarrow+\infty} \dot{V}(t)=0$, which, together with (25), yields $\lim _{t \rightarrow+\infty} \tilde{u}(t)=0, \lim _{t \rightarrow+\infty} \tilde{v}(t)=0$, and $\lim _{t \rightarrow+\infty} \tilde{r}(t)=0$. Then, by following the the above analysis, according to Barbalat's lemma [40], we can further conclude that $\lim _{t \rightarrow+\infty} \dot{\tilde{u}}(t)=0, \lim _{t \rightarrow+\infty} \dot{\tilde{v}}(t)=0$, and $\lim _{t \rightarrow+\infty} \dot{\tilde{r}}(t)=0$. The proof is complete.

\section{REFERENCES}

[1] C. Yang, Z. Li, R. Cui, and B. Xu, "Neural network-based motion control of underactuated wheeled inverted pendulum models," IEEE Trans. Neurl Netw. Learn. Syst., vol. 25, no. 11, pp. 2004-2016, Nov. 2014.

[2] Y.-L. Wang and Q.-L. Han, "Network-based fault detection filter and controller coordinated design for unmanned surface vehicles in network environments," IEEE Trans. Ind. Inform., vol. 12, no. 5, pp. 1753-1765, Oct. 2016.

[3] W. Dong and Y. Guo, "Global time-varying stabilization of underactuated surface vessel," IEEE Trans. Autom. Control, vol. 50, no. 6, pp. 859-864, Jun. 2005.

[4] W. H. Chen, D. J. Ballance, and P. J. Gawthrop, "Optimal control of nonlinear systems: A predictive control approach," Automatica, vol. 39, no. 4, pp. 633-641, Apr. 2003.

[5] Y. Zhang and S. Li, "Time-scale expansion based approximated optimal control for underactuated systems using projection neural networks," IEEE Trans. Syst., Man, Cybern., Syst., vol. 48, no. 11, pp. 1957-1967, Nov. 2018.

[6] M. E. Serrano, G. J. E. Scaglia, S. A. Godoy, V. Mut, and O. A. Ortiz, "Trajectory tracking of underactuated surface vessels: A linear algebra approach," IEEE Trans. Control Syst. Techonol., vol. 22, no. 3, pp. 1103 1111, May 2014.

[7] Z. Yan and J. Wang, "Model predictive control for tracking of underactuated vessels based on recurrent neural networks," IEEE J. Ocean. Eng., vol. 37, no. 4, pp. 717-726, Oct. 2012.

[8] Y. Zhang, S. Li, and X. Liu, "Adaptive near-optimal control of uncertain systems with application to underactuated surface vessels," IEEE Trans. Control Syst. Technol., vol. 26, no. 4, pp. 1204-1218, Jul. 2018.

[9] B. Xu , X. Wang, and Z. Shi, "Robust adaptive neural control of nonminimum phase hypersonic vehicle model," IEEE Trans. Syst., Man, Cybern., Syst., in press, doi: 10.1109/TSMC.2019.2894916.

[10] H. Ashrafiuon, K. R. Muske, L. C. McNinch, and R. A. Soltan, "Slidingmode tracking control of surface vessels," IEEE Trans. Ind. Electron., vol. 55, no. 11, pp. 4004-4012, Nov. 2008.

[11] B. Xu, R. Zhang, S. Li, W. He, and Z. Shi, "Composite neural learning-based nonsingular terminal sliding mode control of MEMS gyroscopes," IEEE Trans. Neural Netw. Learn. Syst., in press, doi: 10.1109/TNNLS.2019.2919931.

[12] R.Yu, Q. Zhu, G. Xia, and Z. Liu, "Sliding mode tracking control of an underactuated surface vessel," IET Control Theory Appl., vol. 6, no. 3, pp. 461-466, Mar. 2012.

[13] L. C. McNinch, H. Ashrafiuon, and K. R. Muske, "Optimal specification of sliding mode control parameters for unmanned surface vessel systems," Proc. ACC, Hyatt Regency Riverfront, St. Louis, MO, USA, Jun., 2009, pp. 2350-2355.

[14] H. Wang, P. Shi, H. Li, and Q. Zhou, "Adaptive neural tracking control for a class of nonlinear systems with dynamic uncertainties," IEEE Trans. Cybern., vol. 47, no. 10, pp. 3075-3087, Oct. 2017.

[15] Y. J. Liu, M. Gong, L. Liu, S. Tong, and C. L. P. Chen, "Fuzzy observer constraint based on adaptive control for uncertain nonlinear MIMO systems with time-varying state constraints," IEEE Trans. Cybern., in press, doi: 10.1109/TCYB.2019.2933700.

[16] Y. J. Liu, Q. Zeng, S. Tong, C. L. P. Chen, and L. Liu, "Actuator failure compensation-based adaptive control of active suspension systems with prescribed performance," IEEE Trans. Ind. Electron., in press, doi: 10.1109/TIE.2019.2937037.

[17] C. Yang, Y. Jiang, Z. Li, W. He, and C. Y. Su, "Neural control of bimanual robots with guaranteed global stability and motion precision," IEEE Trans. Ind. Electron., vol. 13, no. 3, pp. 1162-1171, Jun. 2017.

[18] Z. Zheng and M. Feroskhan, "Path following of a surface vessel with prescribed performance in the presence of input saturation and external disturbances," IEEE Trans. Ind. Electron., vol. 22, no. 6, pp. 2564-2575, Dec. 2017. 
[19] Z. Sun, G. Zhang, B. Yi, and W. Zhang, "Practical proportional integral sliding mode control for underactuated surface ships in the fields of marine practice," Ocean Eng., vol. 142, pp. 217-223, Sep. 2017.

[20] L. Chen, R. Cui, C. Yang, and W. Yan, "Adaptive neural network control of underactuated surface vessels with guaranteed transient performance: Theory and experimental results," IEEE Trans. Ind. Electron., vol. 67, no. 5, pp. 4024-4035, May. 2020.

[21] S. L. Dai, S. He, M. Wang, and C. Yuan, "Adaptive neural control of underactuated surface vessels with prescribed performance guarantees," IEEE Trans. Neural Netw. Learn. Syst., vol. 30, no. 12, pp. 3686-3698, Dec. 2019.

[22] J. X. Zhang and G. H. Yang, "Fault-tolerant fixed-time trajectory tracking control of autonomous surface vessels with specified accuracy," IEEE Trans. Ind. Electron., vol. 67, no. 6, pp. 4889-4899, Jun. 2020.

[23] B. S. Park, J. W. Kwon, and H. Kim, "Neural network-based output feedback control for reference tracking of underactuated surface vessels," Automatica, vol. 77, pp. 353-359, 2017.

[24] D. Chwa, "Global tracking control of underactuated ships with input and velocity constraints using dynamic surface control method," IEEE Trans. Control Syst. Techonol., vol. 19, no. 6, pp. 1357-1370, Nov. 2011.

[25] B. Zhao, D. Liu, and C. Alippi, "Sliding-mode surface-based approximate optimal control for uncertain nonlinear systems with asymptotically stable critic structure," IEEE Trans. Cybern., in press, doi: 10.1109/TCYB.2019.2962011.

[26] B. Luo, D. Liu, H. Wu, D. Wang, and F. L. Lewis, "Policy gradient adaptive dynamic programming for data-based optimal control," IEEE Trans. Cybern., vol. 47, no. 10, pp. 3341-3354, Oct. 2017.

[27] Y. J. Liu, L. Tang, S. Tong, C. L. P. Chen, and D. J. Li, "Reinforcement learning design-based adaptive tracking control with less learning parameters for nonlinear discrete-time MIMO systems," IEEE Trans. Neural Netw. Learn. Syst., vol. 26, no. 1, pp. 165-176, Jan. 2015.

[28] Q. Z, H. Xu, and S. Jagannathan, "Near optimal output feedback control of nonlinear discrete-time systems based on reinforcement neural network learning," IEEE/CAA J. Autom. Sinica, vol. 1, no. 4, pp. 372 384, Oct. 2014.

[29] V. Narayanan and S. Jagannathan, "Event-triggered distributed control of nonlinear interconnected systems using online reinforcement learning with exploration," IEEE Trans. Cybern., vol. 48, no. 9, pp. 2510-2519, Sep. 2018.

[30] Y. Wen, J. Si, X. Gao, and H. Huang, "A new powered lower limb prosthesis control framework based on adaptive dynamic programming," IEEE Trans. Neural Netw. Learn. Syst., vol. 28, no. 9, pp. 2215-2220, Sep. 2017.

[31] A. Sahoo, H. Xu, and S. Jagannathan, "Approximate optimal control of affine nonlinear continuous-time systems using event-sampled neurodynamic programming," IEEE Trans. Neural Netw. Learn. Syst., vol. 28, no. 3, pp. 639-652, Mar. 2017.

[32] G. Y. Tang and D. X. Gao, "Approximation design of optimal controllers for nonlinear systems with sinusoidal disturbances," Nonlinear Ana., Theory Method Appl., vol. 66, no. 2, pp. 403-414, Jan. 2007.

[33] S. Kang, J. Wang, C. Li, and J. Shan, "Nonlinear optimal control with disturbance rejection for asteroid landing," J. Frankl. Inst., vol. 355, no. 16, pp. 8027-8048, Nov. 2018.

[34] K. Abidi and J. X. Xu, "A discrete-time periodic adaptive control approach for time-varying parameters with known periodicity," IEEE Trans. Autom. Control, vol. 53, no. 2, pp. 575-581, Mar. 2008.

[35] J. X. Xu, S. K. Panda, Y. J. Pan, T. H. Lee, and B. H. Lam, "A modular control scheme for PMSM speed control with pulsating torque minimization," IEEE Trans. Ind. Electron., vol. 51, no. 3, pp. 526-536, Jun. 2004.

[36] W. Chen, L. Jiao, R. Li, and J. Li, "Adaptive backstepping fuzzy control for nonlinearly parameterized systems with periodic disturbances," IEEE Trans. Fuzzy Syst., vol. 18, no. 4, pp. 674-685, Aug. 2010.

[37] Y. Wang, L. Zheng, H. Zhang, and W. X. Zheng, "Fuzzy observer-based repetitive tracking control for nonlinear systems," IEEE Trans. Fuzzy Syst., in press, doi: 10.1109/TFUZZ.2019.2936808.

[38] E. Tatlicioglu, N. Cobanoglu, and E. Zergeroglu, "Neural network-based repetitive learning control of euler lagrange systems: An output feedback approach," IEEE Control Syst. Lett., vol. 2, no. 1, pp. 13-18, Jan. 2018.

[39] Y. Zhang, S. Li, S. Kadry, and B. Liao, "Recurrent neural network for kinematic control of redundant manipulators with periodic input disturbance and physical constraints," IEEE Trans. Cybern., vol. 49, no. 12, pp. 4194-4205, Dec. 2019.

[40] H. K. Khalil, Nonlinear Systems. 3nd ed., NJ, USA: Prentice-Hall, 2002. 1993.

[41] K. D. Do, Z. P. Jiang, and J. Pan, "Robust adaptive path following of underactuated ships," Automatica, vol. 40, no. 6, pp. 929-944, Jun. 2004. 